\title{
Estudo de anatomia comparada suína por angiotomografia: contribuições para modelos de pesquisa e treinamento em cirurgia vascular e endovascular
}

\author{
Comparative angiotomographic study of swine vascular anatomy: contributions to \\ research and training models in vascular and endovascular surgery
}

Adenauer Marinho de Oliveira Góes Junior 1,2 (D), Rosa Helena de Figueiredo Chaves (D), Ismari Perini Furlaneto' (D), Emanuelle de Matos Rodrigues ${ }^{1}$ (D), Flávia Beatriz Araújo de Albuquerque ${ }^{1}$ (D), Jacob Hindrik Antunes Smit ${ }^{1}$ (D),

Carolina Pinheiro de Oliveira' (D), Simone de Campos Vieira Abib² (D)

\begin{abstract}
Resumo
Contexto: Modelos com animais de médio e grande porte permitem que pesquisadores avaliem a eficácia e a segurança de procedimentos cardiovasculares em sistemas que se assemelham à anatomia humana e podem ser usados para simular cenários para fins de treinamento. Embora modelos suínos tenham sido extensivamente utilizados, muitos fatores fisiológicos e anatômicos permanecem desconhecidos ou apenas superficialmente descritos. Objetivos: Descrever a anatomia vascular do suíno por tomografia computadorizada, compará-la à anatomia humana e discutir a aplicação dos modelos porcinos em procedimentos abertos e endovasculares. Métodos: Três porcos machos da raça Landrace foram submetidos a tomografia computadorizada. A anatomia vascular de pescoço, tórax, abdome e membros foi analisada e descrita; foram destacadas similaridades e divergências relevantes entre a anatomia vascular de suínos e de humanos e as implicações em procedimentos vasculares nos suínos. Resultados: $O$ território carotídeo, o arco aórtico e os ramos terminais da aorta em suínos apresentaram diferenças marcantes quando comparados aos de humanos. Foram detectadas compressões de veias renal e ilíaca comum, ambas à esquerda, semelhantes às encontradas nas síndromes humanas de Nutcracker e May-Thurner. Medidas vasculares (diâmetro, comprimento e ângulos) de diferentes topografias de suínos foram fornecidas. Conclusões: Os dados fornecidos podem ser úteis para o planejamento de ensaios pré-clínicos e pesquisa básica, bem como para o refinamento do treinamento cirúrgico usando modelos suínos no campo da cirurgia vascular.
\end{abstract}

Palavras-chave: suínos; vasos sanguíneos; procedimentos cirúrgicos vasculares; anatomia comparada; procedimentos endovasculares; angiografia por tomografia computadorizada.

\begin{abstract}
Background: Medium and large animal models allow researchers to evaluate the efficacy and safety of cardiovascular procedures in systems that resemble human anatomy and can be used to simulate scenarios for training purposes. Although porcine models have been used extensively, many physiological and anatomical features remain unknown or only superficially described. Objectives: To describe the normal porcine vascular anatomy on computed tomography scans, compare it to human vascular anatomy, and discuss the application of porcine models for open and endovascular procedures. Methods: Three male Landrace pigs underwent computed tomography. The vascular anatomy of the neck, thorax, abdomen, and limbs was analyzed and described; relevant similarities and differences between porcine and human vascular anatomies and the implications for vascular procedures in pigs are highlighted. Results: The carotid territory, aortic arch, and terminal aorta branches all show marked differences in pigs compared to their human counterparts. Compressions of both left renal and common iliac veins were detected, analogous to those seen in human Nutcracker and May-Thurner syndromes. Vascular measurements (diameters, lengths, and angles) of several different porcine territories are presented. Conclusions: The data presented should be useful for planning preclinical trials and basic research and for refining surgical training using porcine models in vascular fields.
\end{abstract}

Keywords: pigs; blood vessels; vascular surgical procedures; anatomy, comparative; endovascular procedures; computed tomography angiography.

Como citar: Góes Junior AMO, Chaves RHF, Furlaneto IP, et al. Estudo de anatomia comparada suína por angiotomografia: contribuições para modelos de pesquisa e treinamento em cirurgia vascular e endovascular. I Vasc Bras. 2021;20:e20200086. https://doi.org/10.1590/1677-5449.200086

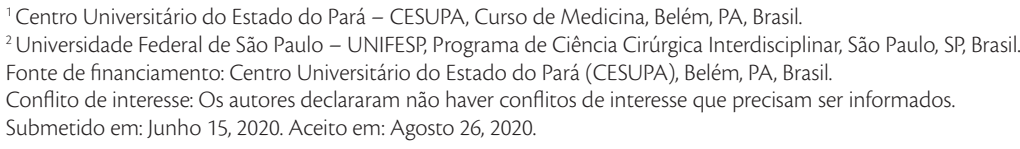

O estudo foi realizado em parceria entre o Programa de Ciência Cirúrgica Interdisciplinar, Universidade Federal de São Paulo (UNIFESP), São Paulo, SP, Brasil e a Faculdade de Medicina, Centro Universitário do Estado do Pará (CESUPA), Belém, PA, Brasil.

\section{Copyright(C) 2021 Os autores. Este é um artigo publicado em acesso aberto (Open Access) sob a licença Creative Commons Attribution, que} permite uso, distribuição e reprodução em qualquer meio, sem restrições desde que o trabalho original seja corretamente citado. 


\section{INTRODUÇÃO}

Doenças cardiovasculares estão entre os principais problemas de saúde e representam tema primordial de pesquisa biomédica ${ }^{1}$. Alguns estudos preliminares foram realizados em animais de menor porte (camundongos, ratos e coelhos), por apresentarem boa relação custo-benefício ${ }^{2}$. Primatas não humanos (por exemplo, babuínos) também são utilizados quando há necessidade de testes com dispositivos maiores, mas o custo e a complexidade das questões éticas são fatores limitantes. Modelos ovinos e suínos representam uma importante opção para pesquisas na área da cirurgia vascular ${ }^{2-4}$. Esses animais permitem aos pesquisadores a avaliação da eficácia e da segurança de procedimentos cardiovasculares em sistemas com anatomia semelhante à humana e que podem ser usados em treinamento ${ }^{1}$.

Há muito tempo suínos são utilizados como modelo cirúrgico, particularmente porque apresentam um preço razoável e sua anatomia e fisiologia cardiovasculares são semelhantes às dos seres humanos ${ }^{1,3-8}$. A anatomia e fisiologia cardíaca suínas foram mais estudadas, como demonstram os estudos sobre distribuição de artérias coronárias, função ventricular, metabolismo cardíaco, eletrofisiologia e desenvolvimento de circulação colateral após infarto agudo do miocárdio ${ }^{1}$.

A recente publicação do genoma e a possibilidade de modificação genética dos suínos aumentaram ainda mais a importância do modelo porcino em pesquisa médica ${ }^{4}$. Um fator chave para o desenvolvimento de modelos experimentais e de treinamento é o conhecimento da anatomia e suas variações ${ }^{5}$. Trabalhos clássicos, baseados em dissecções cirúrgicas, como The anatomy of domestic animals, foram publicados em $1910^{9}$, seguidos por outros que descreveram o uso de suínos para uma variedade de procedimentos ${ }^{1,2,5-8,10-16}$

Nas últimas décadas, houve aumento na complexidade das cirurgias abertas e endovasculares. Métodos de imagem não invasivos, como tomografia computadorizada (TC), tornaram-se rotineiros no planejamento terapêutico e no acompanhamento de casos, e estão progressivamente substituindo a angiografia tradicional. Apesar dos avanços técnicos, há carência de descrições detalhadas da anatomia vascular extracardíaca de suínos ${ }^{3,5,6,13}$.

O objetivo deste estudo foi descrever as características da anatomia vascular de suínos por TC, compará-la à anatomia humana e discutir a aplicação de modelos porcinos para procedimentos cirúrgicos abertos e endovasculares.

\section{MÉTODOS}

Este estudo foi aprovado pelo comitê institucional de ética em experimentação animal.

Três porcos machos da raça Landrace foram utilizados para a aquisição de imagens de TC. Seus pesos eram 45,4 kg, 49,2 kg e 52,3 kg. Os animais foram mantidos em alojamento padrão (controle de temperatura e umidade) e permaneceram em jejum 12 horas antes dos exames.

\section{Protocolo anestésico}

O procedimento foi realizado sob anestesia geral e monitoramento por médico veterinário. Para prémedicação, foram utilizadas injeção intramuscular de cetamina $(15 \mathrm{mg} / \mathrm{kg})$ e xilazina $(1,5 \mathrm{mg} / \mathrm{kg})$.

Indução anestésica se deu por injeção intravenosa de propofol $(2,5-5 \mathrm{mg} / \mathrm{kg})$; a manutenção foi realizada com infusão contínua do mesmo medicamento $(0,1-0,2 \mathrm{mg} / \mathrm{kg} / \mathrm{min})$. Os animais foram posicionados em decúbito ventral durante a aquisição das imagens. Não houve necessidade de intubação.

\section{Tomografia computadorizada}

As imagens foram obtidas em um tomógrafo de 64 canais, com cortes de $0,625 \mathrm{~mm}$. O contraste iodado endovenoso utilizado foi o loexol $(120 \mathrm{~mL}$ em velocidade de fluxo de $5 \mathrm{~mL} / \mathrm{s}$ ). As medidas vasculares foram obtidas na fase arterial pela técnica de bolus-tracking, e a fase venosa foi iniciada imediatamente após a arterial.

Após a realização da TC, os animais foram utilizados no ensino de habilidades cirúrgicas na instituição e, em seguida, submetidos a eutanásia por injeção intravenosa de cloreto de potássio.

\section{Análise das imagens}

Utilizou-se a plataforma Horos ${ }^{\mathrm{TM}} \mathrm{V} 3.1 .0$ (Horosproject. org). Para descrições anatômicas, os termos "ventral" e "dorsal" foram adotados como correspondentes a "anterior" e "posterior" em humanos. Da mesma forma, "cranial" e "caudal" foram utilizados como equivalentes a "superior" e "inferior"

Os maiores diâmetros dos vasos foram medidos em dois eixos perpendiculares, obtendo-se a seguir o diâmetro médio naquela topografia.

As medidas foram feitas em marcos anatômicos relacionados a procedimentos cirúrgicos e endovasculares, como angioplastias viscerais, implante de filtro de veia cava e endopróteses de aorta (Figura 1).

\section{Análise estatística}

Utilizou-se o software Prism 6 (Graphpad Software, La Jolla, EUA). Foram determinadas média, mediana, desvio padrão e coeficiente de variação $(\mathrm{CV})$. 


\section{RESULTADOS}

\section{Anatomia arterial}

Em relação às carótidas, as externas apresentaram diâmetro maior que as internas.

A aorta apresentou estreitamento acentuado e progressivo desde sua origem até sua porção distal, com diâmetro médio de $25 \mathrm{~mm}$ na aorta ascendente e de $9,8 \mathrm{~mm}$ na aorta terminal.

Entre os ramos viscerais, a artéria mesentérica cranial apresentou maior diâmetro, seguida pela artéria celíaca; a mesentérica caudal teve o menor diâmetro. Os diâmetros arteriais são mostrados na Tabela 1.
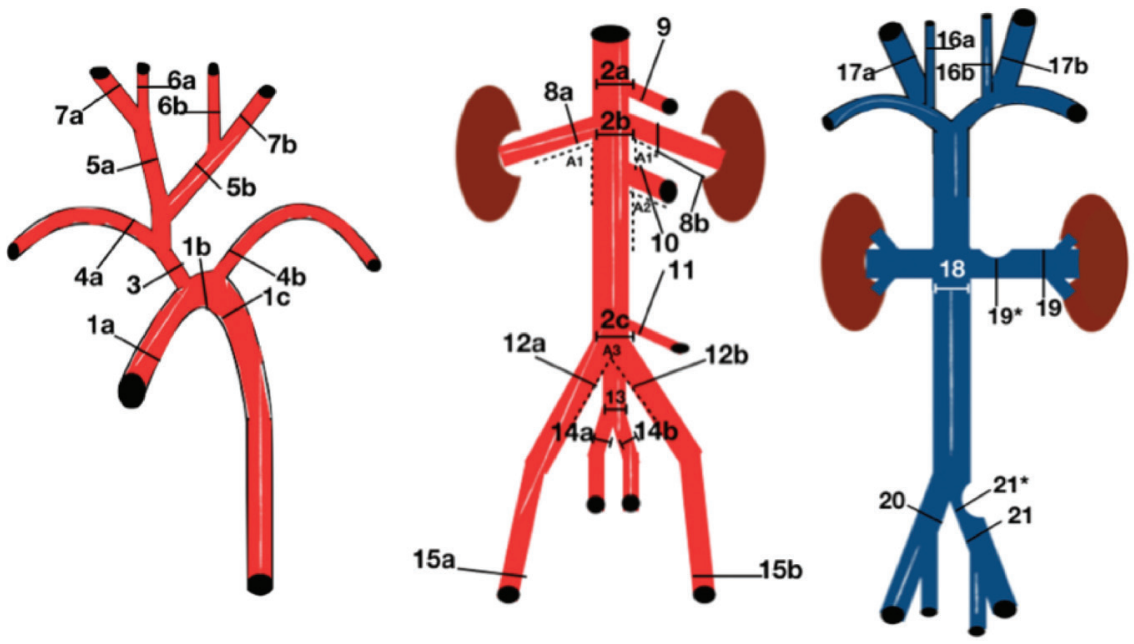

Figura 1. llustração dos pontos de referência para as medidas. *: ponto de aferição do diâmetro venoso no ponto de compressão.

Tabela 1. Diâmetros arteriais.

\begin{tabular}{|c|c|c|c|c|c|}
\hline Diâmetro arterial & Mínimo & Máximo & Média & DP & CV\% \\
\hline Aorta ascendente (1a) & 22,9 & 28,2 & 25,0 & 2,8 & 11,1 \\
\hline Arco aórtico (1b) & 18,7 & 24,1 & 21,5 & 2,7 & 12,5 \\
\hline Aorta descendente (1c) & 16,4 & 22,5 & 19,4 & 3,0 & 15,6 \\
\hline Aorta a (2a) & 12,1 & 13,4 & 12,9 & 0,7 & 5,4 \\
\hline Aorta abdominal (2b) & 8,7 & 11,1 & 10,2 & 1,3 & 12,6 \\
\hline Aorta abdominal $(2 \mathrm{c})$ & 9,1 & 10,8 & 9,8 & 0,9 & 9,1 \\
\hline Tronco braquiocefálico (3) & 10,6 & 13,0 & 11,5 & 1,3 & 11,3 \\
\hline Artéria subclávia direita (4a) & 4,3 & 6,8 & 5,8 & 1,4 & 23,5 \\
\hline Artéria subclávia esquerda (4b) & 4,4 & 6,2 & 5,6 & 1,1 & 19,1 \\
\hline Carótida comum direita ( $5 \mathrm{a}$ ) & 4,0 & 5,6 & 4,8 & 0,8 & 17,0 \\
\hline Carótida comum esquerda (5b) & 4,7 & 5,4 & 4,9 & 0,4 & 7,8 \\
\hline Carótida interna direita (6a) & 3,5 & 4,0 & 3,8 & 0,3 & 7,7 \\
\hline Carótida interna esquerda (6b) & 3,4 & 3,7 & 3,5 & 0,2 & 5,0 \\
\hline Carótida externa direita (7a) & 4,5 & 5,5 & 5,0 & 0,5 & 10,6 \\
\hline Carótida externa esquerda (7b) & 5,1 & 5,8 & 5,4 & 0,4 & 6,8 \\
\hline Artéria renal direita (8a) & 3,5 & 5,1 & 4,4 & 0,8 & 18,9 \\
\hline Artéria renal esquerda (8b) & 5,1 & 5,7 & 5,3 & 0,4 & 6,7 \\
\hline Tronco celíaco (9) & 4,8 & 7,1 & 6,2 & 1,2 & 19,6 \\
\hline Artéria mesentérica cranial (10) & 6,9 & 8,2 & 7,4 & 0,7 & 9,8 \\
\hline Artéria mesentérica caudal (11) & 2,0 & 2,6 & 2,4 & 0,3 & 13,6 \\
\hline Artéria ilíaca externa direita (12a) & 5,4 & 8,2 & 7,2 & 1,6 & 22,0 \\
\hline Artéria ilíaca externa esquerda (12b) & 6,1 & 7,3 & 6,7 & 0,6 & 8,6 \\
\hline Tronco ilíaco interno (13) & 4,7 & 8,0 & 6,1 & 1,7 & 28,4 \\
\hline Artéria ilíaca interna direita (14a) & 3,7 & 4,7 & 4,3 & 0,6 & 12,7 \\
\hline Artéria ilíaca interna esquerda (14b) & 3,8 & 4,3 & 4,1 & 0,3 & 6,4 \\
\hline Artéria femoral superficial direita (15a) & 4,9 & 6,4 & 5,8 & 0,8 & 13,7 \\
\hline Artéria femoral superficial esquerda (15b) & 4,8 & 5,6 & 5,3 & 0,4 & 8,2 \\
\hline
\end{tabular}

$\mathrm{DP}=$ desvio padrão; $\mathrm{CV}$ = coeficiente de variação; números e letras entre parênteses (por exemplo, 1a) correspondem aos pontos de medidas na Figura 1. 
Um animal apresentou artéria renal esquerda mais caudal que a direita; nos outros dois animais, ambas as renais emergiam no mesmo nível da aorta. Os ângulos das artérias renais em relação à aorta diferiram amplamente, de $82,5^{\circ}$ a $114,5^{\circ}$ (média de $96,8^{\circ}$ ) à direita e de $76,8^{\circ}$ a $116,3^{\circ}$ (média de $93,8^{\circ}$ ) à esquerda.

O comprimento da artéria femoral superficial direita (na altura da cabeça do fêmur) até a aorta terminal (L1) foi em média de $12,9 \mathrm{~cm}$. A partir do mesmo ponto, o comprimento médio foi de $22,1 \mathrm{~cm}$ até a artéria renal mais caudal (L2), $30 \mathrm{~cm}$ até a artéria celíaca (L3), e 58,9 cm até a artéria subclávia esquerda (L4). Ângulos arteriais e comprimentos estão dispostos na Tabela 2.

\section{Diâmetros venosos}

As jugulares externas apresentaram diâmetro muito maior que as internas. $\mathrm{O}$ diâmetro médio da jugular externa direita foi $9,2 \mathrm{~mm}$, enquanto que o da esquerda foi de $8,6 \mathrm{~mm}$; já a jugular interna apresentou diâmetro médio de $4 \mathrm{~mm}$ no lado direito e 4,5 $\mathrm{mm}$ no lado esquerdo.

O diâmetro médio da veia cava caudal, proximalmente ao nível da veia renal mais caudal, foi de $10,5 \mathrm{~mm}$.
Devido à detecção de estreitamento na veia renal esquerda entre a aorta e a artéria mesentérica cranial (semelhante à síndrome de Nutcracker), as medidas foram feitas no hilo renal (diâmetro médio de 5,8 $\mathrm{mm}$ ) e no ponto de compressão máxima (diâmetro médio de $2,8 \mathrm{~mm})$.

O diâmetro médio da veia ilíaca comum direita foi de 7,2 mm; o diâmetro médio da veia ilíaca comum esquerda foi de $6,9 \mathrm{~mm}$ proximalmente à confluência com a cava e 2,6 mm no ponto de compressão máxima entre a aorta e as vértebras adjacentes (semelhante à síndrome de May-Thurner). Diâmetros venosos são exibidos na Tabela 3.

\section{Coeficientes de variação}

As maiores variações (> 20\%) foram detectadas nos seguintes pontos: veia renal esquerda no ponto de compressão máxima $(\mathrm{CV}=29,6 \%)$, tronco ilíaco interno $(\mathrm{CV}=28,4 \%)$, artéria subclávia direita $(\mathrm{CV}=23,5 \%)$, artéria ilíaca externa direita $(\mathrm{CV}=22,0 \%)$ e ângulo de emergência da artéria renal esquerda $(\mathrm{CV}=21,7 \%)$.

Algumas informações anatômicas relacionadas à anatomia arterial e venosa são ilustradas nas Figuras 2 a 6.

Tabela 2. Ângulos e comprimentos arteriais.

\begin{tabular}{lccccc}
\hline \multicolumn{1}{c}{ Ângulos/comprimento arterial } & Mínimo & Máximo & Média & DP & CV\% \\
\hline Ângulo da artéria renal direita (A1) & 82,5 & 114,5 & 96,8 & 16,3 & 16,8 \\
Ângulo da artéria renal esquerda (A1 ${ }^{*}$ ) & 76,8 & 116,3 & 93,8 & 20,3 & 21,7 \\
Ângulo aórtico-mesentérico (A2) & 64,5 & 87,2 & 74,8 & 11,5 & 15,4 \\
Ângulo das artérias ilíacas externas (A3) & 31,7 & 44,0 & 36,6 & 6,5 & 17,8 \\
Comprimento aórtico-femoral (L1) & 12,4 & 13,5 & 12,9 & 0,5 & 4,0 \\
Comprimento femoral-renal (L2) & 19,9 & 23,6 & 22,1 & 1,9 & 8,6 \\
Comprimento femoral-celíaca (L3) & 27,0 & 31,6 & 30,0 & 2,6 & 8,6 \\
Comprimento femoral-subclávia (L4) & 52,9 & 62,0 & 58,9 & 5,2 & 8,9 \\
\hline
\end{tabular}

$\mathrm{DP}=$ desvio padrão; $\mathrm{CV}=$ coeficiente de variação; números e letras entre parênteses (por exemplo, A1) correspondem aos pontos de medidas na Figura 1. L1, L2, L3 e L4 representam os comprimentos entre a artéria femoral superficial (na altura da cabeça do fêmur) e os seguintes pontos: aorta terminal, artéria renal mais caudal, artéria celíaca e artéria subclávia esquerda, respectivamente.

Tabela 3. Diâmetros venosos.

\begin{tabular}{|c|c|c|c|c|c|}
\hline Diâmetro venoso & Mínimo & Máximo & Média & $\mathrm{DP}$ & CV\% \\
\hline Jugular interna direita (16a) & 3,6 & 4,3 & 4,0 & 0,4 & 9,2 \\
\hline Jugular interna esquerda (16b) & 3,8 & 5,4 & 4,5 & 0,8 & 17,2 \\
\hline Jugular externa direita (17a) & 8,0 & 10,5 & 9,2 & 1,3 & 13,8 \\
\hline Jugular externa esquerda (17b) & 7,7 & 9,2 & 8,6 & 0,8 & 9,5 \\
\hline Veia cava caudal (18) & 9,2 & 11,7 & 10,5 & 1,2 & 11,8 \\
\hline Veia renal esquerda [H] (19) & 5,3 & 6,4 & 5,8 & 0,5 & 9,4 \\
\hline Veia renal esquerda $[C P]\left(19^{*}\right)$ & 1,8 & 3,4 & 2,8 & 0,8 & 29,6 \\
\hline Veia ilíaca comum direita (20) & 6,5 & 7,9 & 7,2 & 0,7 & 10,2 \\
\hline Veia ilíaca comum esquerda (21) & 6,6 & 7,4 & 6,9 & 0,4 & 5,7 \\
\hline Veia ilíaca comum esquerda [CP] $\left(21^{*}\right)$ & 2,3 & 2,8 & 2,6 & 0,3 & 11,0 \\
\hline
\end{tabular}

$\mathrm{DP}$ = desvio padrão; $\mathrm{CV}=$ coeficiente de variação; $\mathrm{H}$ = hilo renal; $\mathrm{CP}=$ ponto de compressão; números e letras entre parênteses (por exemplo, 16a) correspondem aos pontos medidos na Figura 1. 


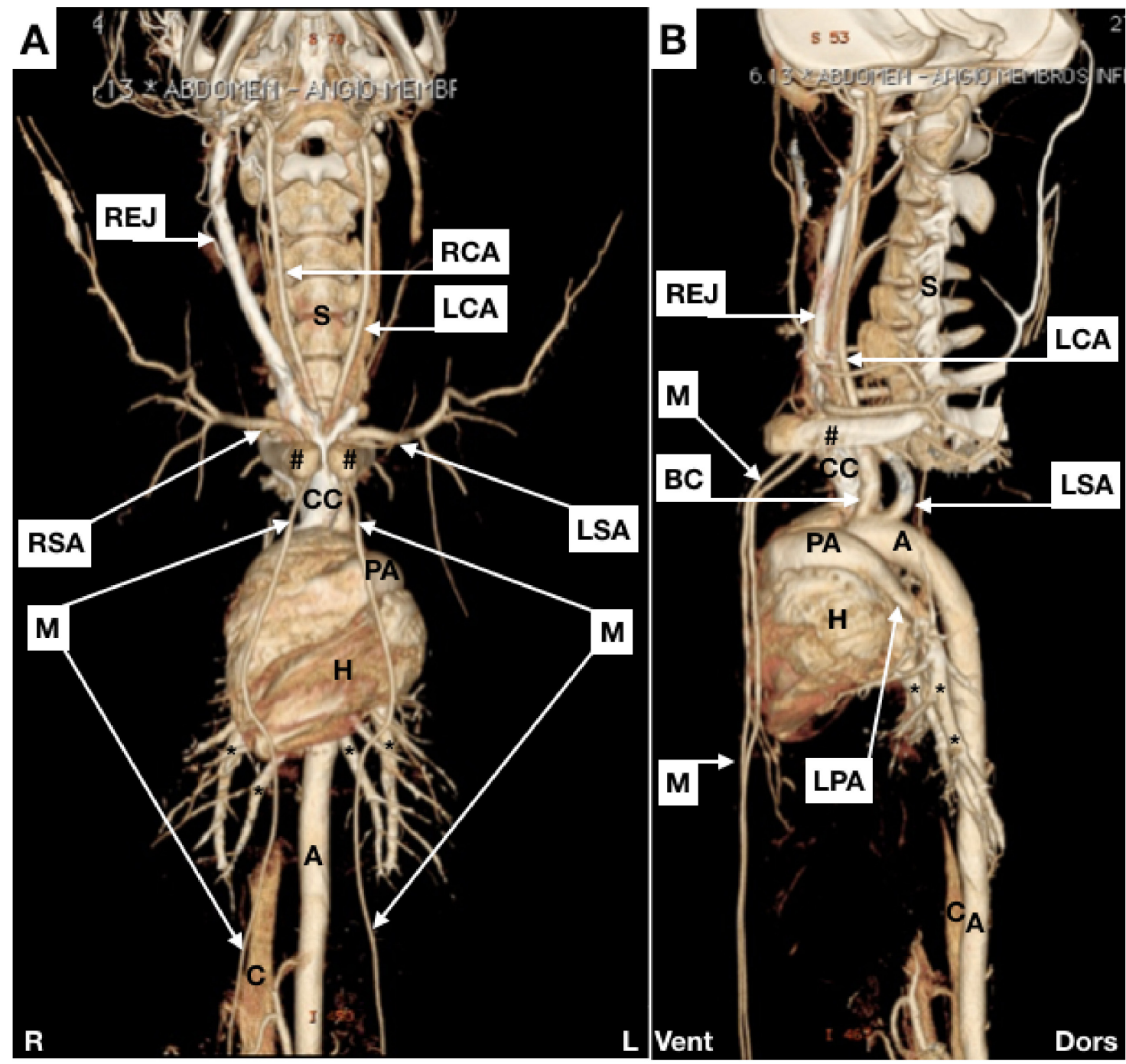

Figura 2. Imagens em projeção de intensidade máxima (maximal intensity projection, MIP). (A) visão frontal; (B) visão lateral. $\mathrm{REJ}=$ veia jugular externa direita; $\mathrm{RCA}=$ carótida comum direita; $\mathrm{LCA}$ = carótida comum esquerda; $\mathrm{S}$ = coluna vertebral; \# = clavículas; $C C$ = veia cava cranial; $R S A$ = artéria subclávia direita; $L S A=$ artéria subclávia esquerda; $M=$ artérias mamárias; $P A=$ artéria pulmonar (tronco); $\mathrm{H}=$ coração; $\mathrm{A}=$ aorta; $\mathrm{C}=$ veia cava caudal; $\mathrm{R}=$ direita; $\mathrm{L}=$ esquerda; $\mathrm{BC}=$ artéria braquiocefálica; $\mathrm{LPA}=$ artéria pulmonar esquerda; ${ }^{*}=$ ramos das artérias pulmonares; Vent = ventral; Dors = dorsal.

\section{Anatomia suína $\times$ humana}

O arco aórtico porcino dá origem a dois ramos: artérias braquiocefálica e subclávia esquerda; em humanos, essa estrutura origina, normalmente, os três ramos supra-aórticos. A partir da artéria braquiocefálica, emergem a artéria subclávia direita e o tronco bicarotídeo, que origina as carótidas comuns (Figura 2).

Outra disparidade ocorre na bifurcação carotídea. Em humanos, a artéria carótida comum apresenta um bulbo e bifurca-se em carótidas interna e externa. Já em suínos, a artéria carótida externa emerge como continuidade da carótida comum e é mais calibrosa que a interna.
Apesar de não fazer parte do objetivo desta pesquisa descrever a anatomia da região cefálica, destacam-se diferenças em comparação à circulação humana, como a presença de múltiplas comunicações extracranianas entre as artérias carótidas externa e interna e da rete mirabile, uma complexa rede arteriolar epidural com formato ovoide $2,6,9,18$.

No que diz respeito às singularidades venosas, divegindo dos humanos, a jugular externa é quase tão profunda quanto a interna, porém está localizada em um compartimento diferente. Também é muito mais calibrosa que a interna, a qual cursa paralela à carótida (Figura 3A). Além disso, as jugulares internas e externas de cada lado se fundem à veia 

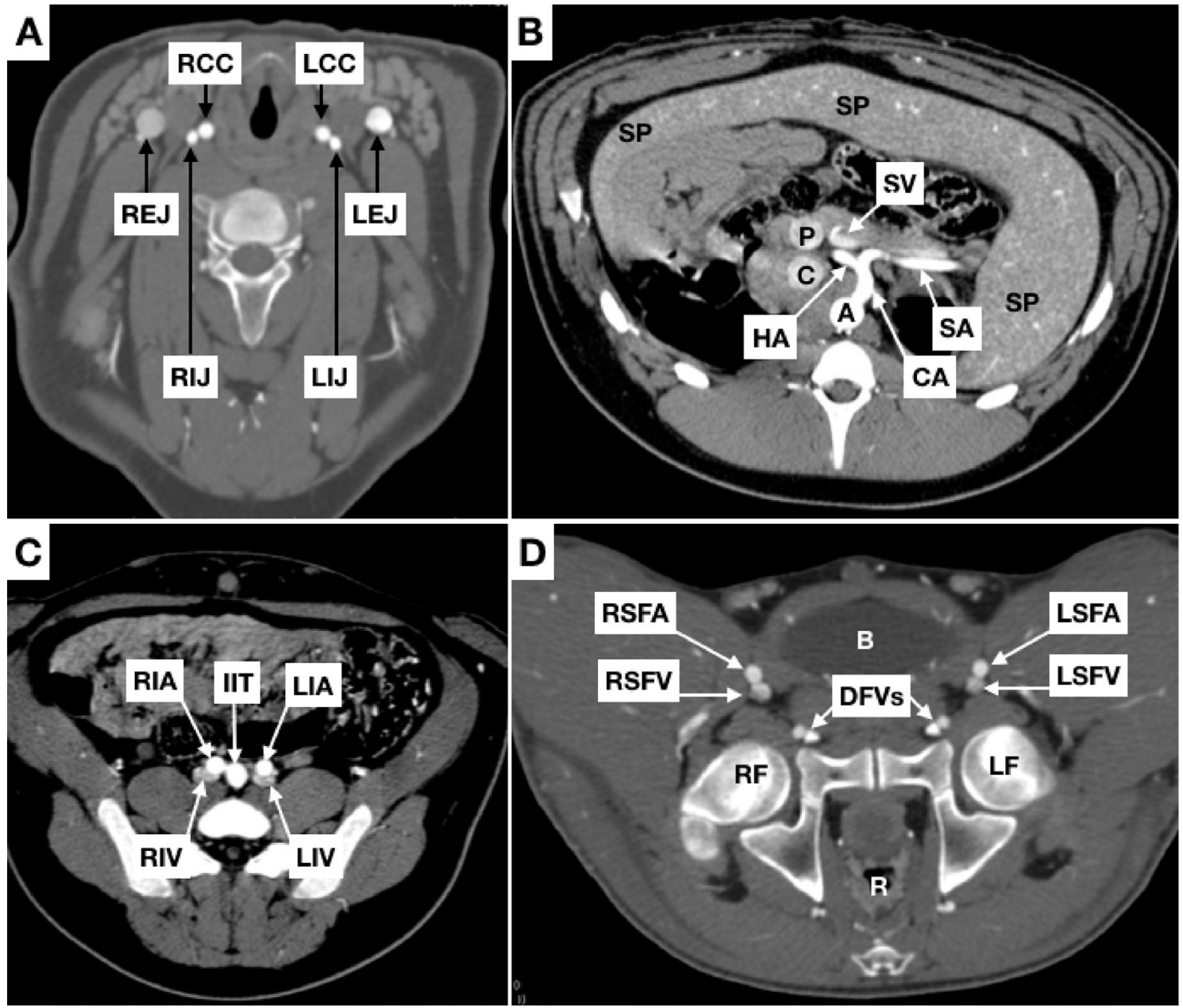

Figura 3. Cortes coronais. (A) pescoço; (B) abdome; (C) e (D) pelve. RCC = carótida comum direita; LCC = carótida comum esquerda; REJ = veia jugular externa direita; $L E J$ = veia jugular externa esquerda; RIJ = veia jugular interna direita; $L I J=$ veia jugular interna esquerda; $\mathrm{A}=$ aorta; $\mathrm{C}=$ veia cava caudal; $\mathrm{P}=$ veia porta; $\mathrm{CA}=$ artéria celíaca; $\mathrm{SA}=$ artéria esplênica; $\mathrm{HA}=$ artéria hepática; SV = veia esplênica; SP = baço; RIA = artéria ilíaca externa direita; LIA = artéria ilíaca externa esquerda; RIV = veia ilíaca comum direita; LIV = veia ilíaca comum esquerda; IIT = tronco ilíaco interno; RSFA = artéria femoral superficial direita; LSFA = artéria femoral superficial esquerda; RSFV = veia femoral superficial direita; LSFV = veia femoral superficial esquerda; DFVs = vasos femorais profundos (artérias e veias); $B$ = bexiga; $R$ = reto; $R F=$ fêmur direito; $L F=$ fêmur esquerdo.

subclávia, formando um tronco curto que, ao se unir ao contralateral equivalente, origina a cava cranial.

O segmento torácico da cava caudal é muito mais longo que o equivalente no humano (segmento torácico da veia cava inferior) e possui uma porção circundada por parênquima pulmonar. A cava caudal atravessa o diafragma e recebe as veias hepáticas, semelhante ao segmento retro-hepático da cava inferior humana (Figura 4B).

Na veia cava caudal desembocam as veias renais. Suínos podem apresentar um importante estreitamento na veia renal esquerda, entre a aorta abdominal e a artéria mesentérica cranial, análogo à síndrome de Nutcracker em humanos ${ }^{19-21}$ (Figuras 4C e 4D).
A aorta abdominal emite as artérias celíaca, mesentéricas cranial e caudal, e renais direita e esquerda, em diferentes ângulos (Figuras 5A e 5D).

Suínos não possuem artéria ilíaca "comum", pois a aorta termina em "trifurcação", emitindo artérias ilíacas externas direita e esquerda e o tronco ilíaco interno, que por sua vez origina as artérias ilíacas internas direita e esquerda e a artéria sacral mediana (Figuras 5B, 6A e 6B).

A artéria ilíaca externa continua como artéria femoral. De forma divergente ao observado em humanos, a artéria femoral (equivalente à femoral comum em humanos), ainda intrapélvica, ramifica-se em artérias femorais superficial e profunda; a artéria na projeção 

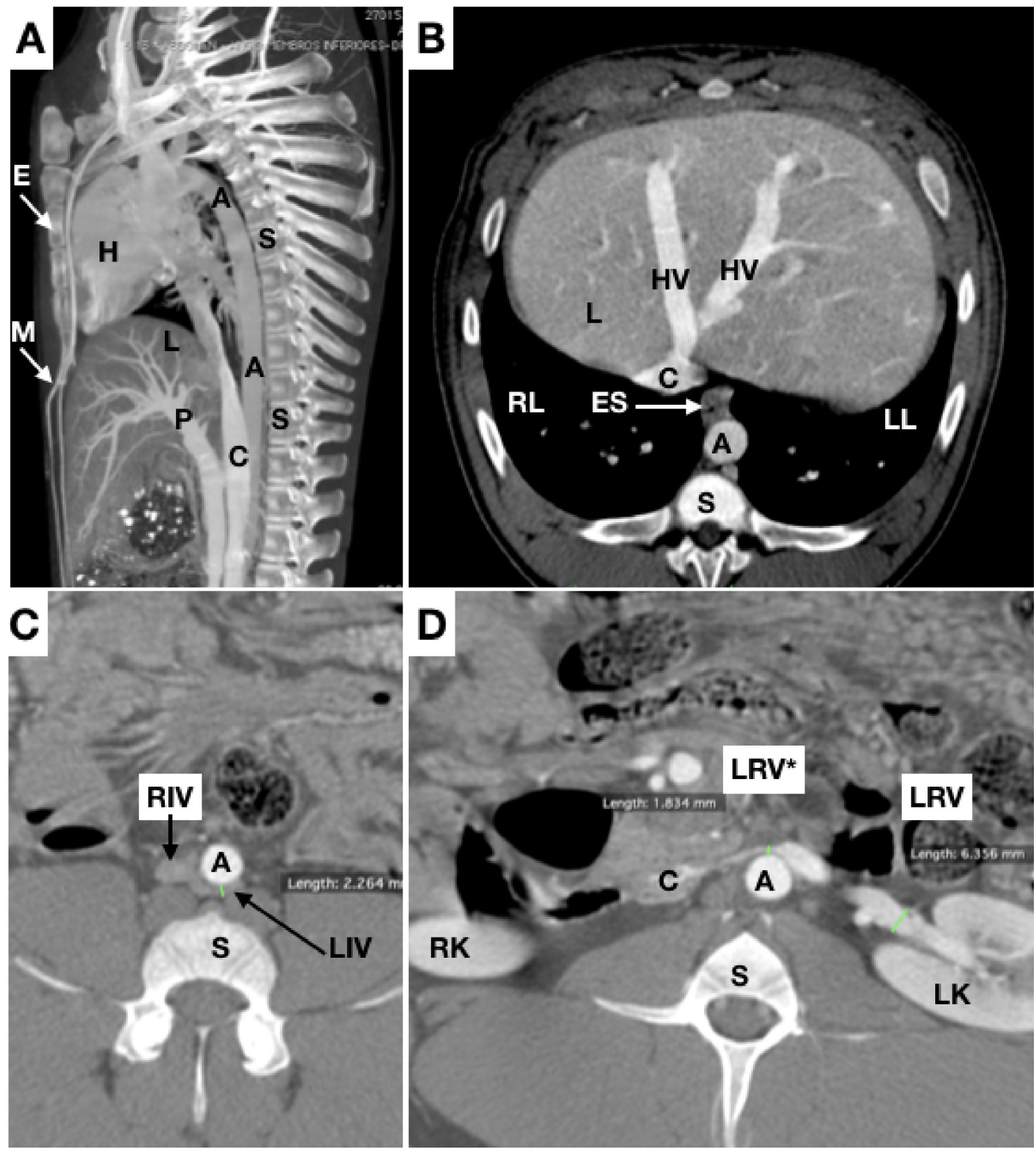

Figura 4. (A) corte sagital; (B), (C) e (D) cortes axiais. $A=$ aorta; $C$ = veia cava caudal; $P$ = veia porta; $L$ = fígado; $S=$ coluna vertebral; $\mathrm{E}=$ esterno; $\mathrm{HV}$ = veia hepática; $M$ = artérias mamárias; $\mathrm{H}=$ coração; $E S$ = esôfago; $\mathrm{RL}$ = pulmão direito; $\mathrm{LL}=$ pulmão esquerdo; $L R V=$ veia renal esquerda; $L R V^{*}=$ comprimido entre a aorta e a artéria mesentérica cranial; $R I V=$ veia ilíaca comum direita; LIV = veia ilíaca comum; RK = rim direito; LK = rim esquerdo.

da cabeça do fêmur é a femoral superficial, enquanto que os vasos femorais profundos percorrem um trajeto próximo ao púbis (Figuras 3D e 6C).

A anatomia venosa pélvica assemelha-se à humana: as veias ilíacas interna e externa fundem-se em veias ilíacas comuns, que confluem formando a veia cava caudal. Detectamos ocorrência de compressões da veia ilíaca comum esquerda, análogas à síndrome de May-Thuner; contudo, nos suínos o estreitamento venoso ocorre entre a aorta e a coluna vertebral, e não entre a artéria ilíaca comum direita e a coluna vertebral, como em humanos ${ }^{22,23}$ (Figura 4C).

$\mathrm{O}$ suprimento arterial dos membros posteriores diverge bastante daquele equivalente em humanos. A artéria femoral superficial, no terço médio do fêmur, divide-se nas artérias safena e poplítea. A artéria safena 


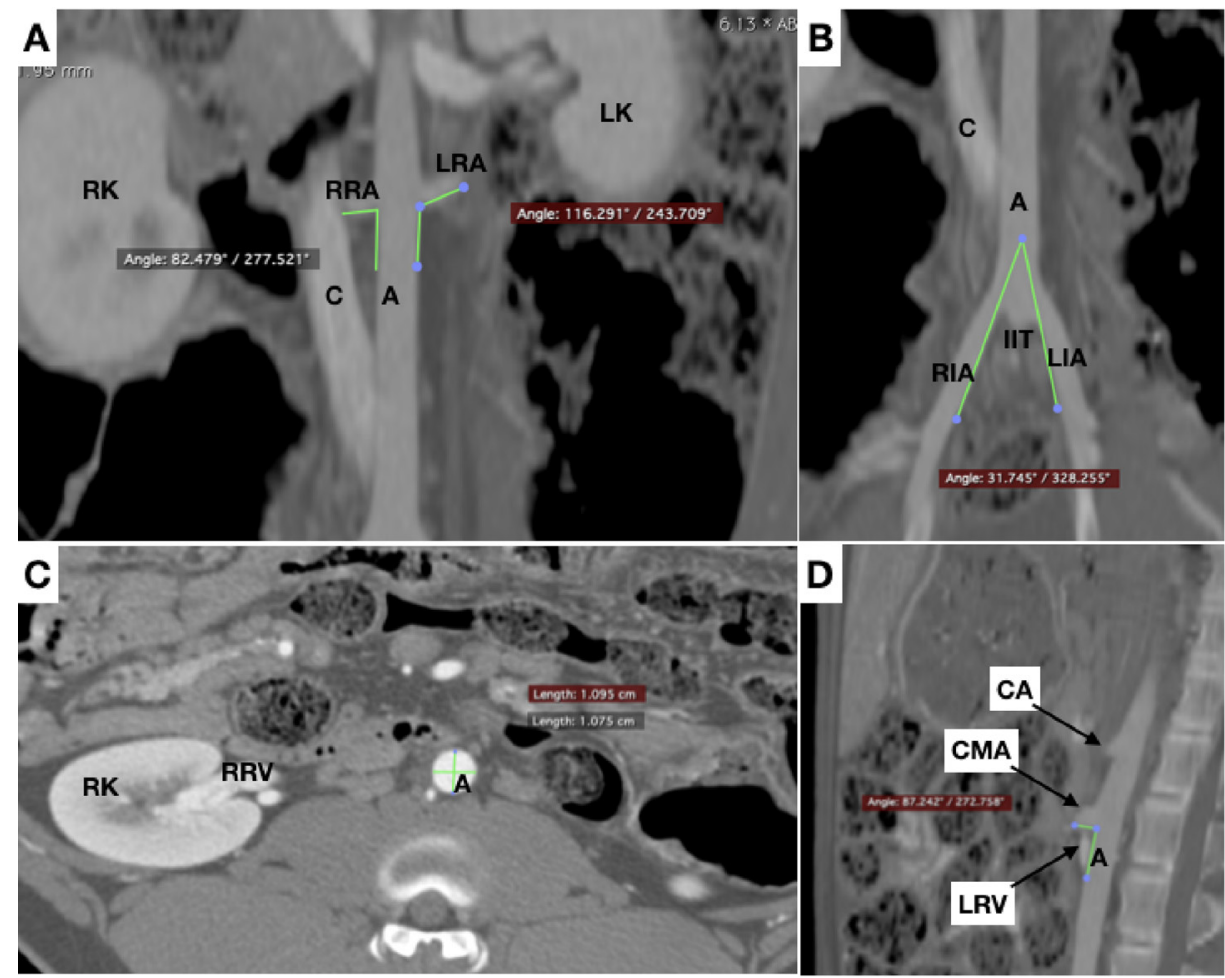

Figura 5. (A) e (B) Cortes coronais, aferição dos ângulos entre as artérias renais e a aorta e entre as artérias ilíacas externas (trifurcação da aorta), respectivamente; (C) Corte axial para medir diâmetro aórtico; (D) Corte sagital para medir o ângulo entre a aorta e a artéria mesentérica anterior. $\mathrm{A}=$ aorta; $\mathrm{C}=$ veia cava caudal; $\mathrm{RRA}=$ artéria renal direita; $\mathrm{LRA}=$ artéria renal esquerda; $\mathrm{RK}=$ rim direito; $L K=$ rim esquerdo; RIA = artéria ilíaca externa direita; LIA = artéria ilíaca externa esquerda; IIT = tronco ilíaco interno; $R R V=$ veia renal direita; $C A$ = artéria celíaca; $C M A$ = artéria mesentérica cranial; $L R V=$ veia renal esquerda.

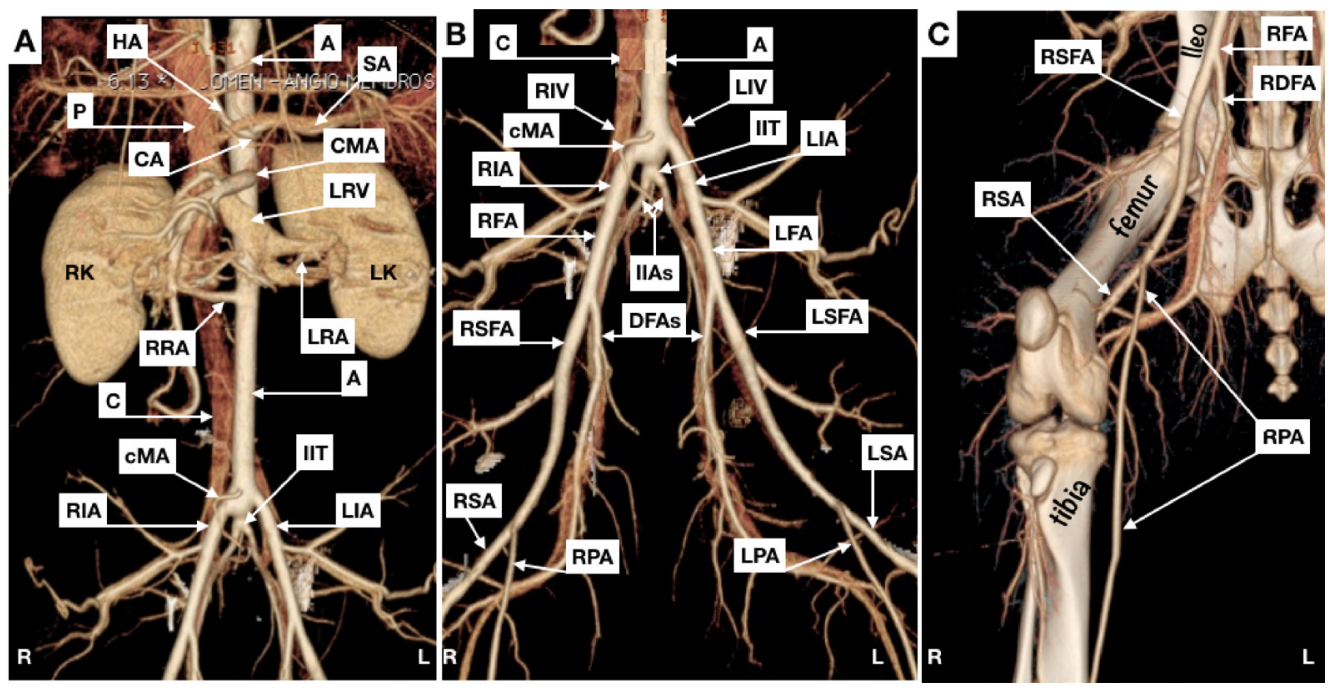

Figura 6. Imagens em projeção de intensidade máxima (maximal intensity projection, MIP). (A) abdome (visão frontal); (B) pelve (visão frontal); C) membro direito posterior (visão frontal). $\mathrm{HA}=$ artéria hepática; $\mathrm{A}=$ aorta; $\mathrm{SA}=$ artéria esplênica (parcialmente sobreposta pela veia esplênica); $P=$ veia porta; $C A=$ artéria celíaca; $C M A=$ artéria mesentérica cranial; $L R V=$ veia renal esquerda; $\mathrm{RK}=$ rim direito; $\mathrm{LK}=$ rim esquerdo; $\mathrm{RRA}=$ artéria renal direita; $\mathrm{LRA}=$ artéria renal esquerda; $\mathrm{C}=$ veia cava caudal; $\mathrm{CMA}=$ artéria mesentérica caudal; IIT = tronco ilíaco interno; RIA = artéria ilíaca externa direita; LIA = artéria ilíaca externa esquerda; $R=$ direita; $\mathrm{L}$ = esquerda; $\mathrm{RIV}=$ veia ilíaca comum direita; $\mathrm{LIV}=$ veia ilíaca comum esquerda; RFA = artéria femoral comum direita; $L F A=$ artéria femoral comum esquerda; IIAs = artérias ilíacas internas; RSFA = artéria femoral superficial direita; DFAs = artérias femorais profundas (parcialmente sobrepostas pela veia femoral profunda); LSFA = artéria femoral superficial esquerda; RSA = artéria safena direita; $\mathrm{RPA}=$ artéria poplítea direita; $\mathrm{LPA}=$ artéria poplítea esquerda; $\mathrm{LSA}=$ artéria safena esquerda; $R D F A=$ artéria femoral profunda direita. 
emite ramos que suprem grande parte da musculatura, inclusive as artérias digitais plantares e dorsais. A artéria poplítea se anterioriza através da membrana interóssea, passando a ser denominada artéria tibial cranial e fornecendo vários ramos musculares.

\section{DISCUSSÃO}

\section{Modelos suínos para treinamento e experimentação}

Animais de experimentação são frequentemente utilizados para avaliar a eficácia e a segurança de novos tratamentos antes da aplicação em ensaios clínicos ${ }^{1-7,9-15,24}$. Embora suínos tenham sido extensivamente utilizados em várias áreas, como genômica, transplante de órgãos, oncologia, trauma, doenças neurológicas e cardiovasculares $^{2-4,10,18}$, muitos fatores fisiológicos e anatômicos importantes continuam desconhecidos ou superficialmente descritos ${ }^{1,5,25}$.

A literatura apresenta várias descrições anatômicas discordantes, como a da "bifurcação" da aorta", que em suínos é, na verdade, uma "trifurcação". Além disso, as artérias subclávias ${ }^{3,6,11,15,27}$, que surgem da artéria braquiocefálica no lado direito e do arco aórtico no lado esquerdo, suprindo os membros anteriores do animal, são ocasionalmente chamadas de artérias braquiais ${ }^{9}$; outro exemplo são as artérias mesentéricas anterior e posterior ${ }^{9}$, para as quais também se encontram descrições como superior ${ }^{3,11,28}$ e inferior ${ }^{3,11,15,28}$ ou cranial e caudal ${ }^{6}$.

Diferentes raças têm sido usadas em pesquisa vascular. Porcos Yucatan foram utilizados para estudos envolvendo cateteres de hemodiálise, pois essa raça para de crescer após 1 ano de idade, com peso médio de $70 \mathrm{~kg}$, tornando-os apropriados para esse propósito ${ }^{13}$. Essa mesma raça, aos 3 meses de idade, com peso entre 32,4 e $34,5 \mathrm{~kg}$, foi utilizada para anastomoses vasculares microcirúrgicas em um modelo de transplante de tíbia'; e, com 4 meses de idade, foi usada para avaliar a diferença entre os gêneros, em relação aos resultados de stents coronarianos ${ }^{24}$.

A raça de miniporco Göttingen foi utilizada em estudos de anatomia vascular por tomografia, mais especificamente com enfoque em problemas pediátricos, visto que, com 11 semanas de vida, esses animais têm o peso aproximado do ser humano ao nascer ${ }^{3}$, com um perfil adequado para esses projetos.

Porcos da raça Landrace, a mesma utilizada no presente estudo, pesando aproximadamente $55 \mathrm{~kg}$, foram utilizados no desenvolvimento experimental de aneurismas de aorta e no tratamento de endoleaks tipos IA e II por laparoscopia e toracoscopia ${ }^{13}$. Com peso entre 70 e $95 \mathrm{~kg}$, foram usados para pesquisa com oclusão ressuscitativa por balão endovascular da aorta (resuscitative endovascular balloon occlusion of the aorta, REBOA $)^{29}$. Suínos Landrace com peso entre 20 e $30 \mathrm{~kg}$ também foram utilizados com sucesso para o treinamento de cirurgia aórtica por videolaparoscopia ${ }^{26}$. Em 1998, um estudo de anatomia radiológica feito em porcos Landrace pesando entre 20 e $25 \mathrm{~kg}$ descreveu aspectos angiográficos de cérebro, cabeça, pescoço, tórax, abdome e pelve ${ }^{6}$.

A opção por estudar essa raça, além de esta já ter sido utilizada em treinamentos e pesquisas de várias técnicas cirúrgicas abertas e endovasculares, se deve ao fato de a raça estar facilmente disponível e ser adequada a uma variedade de procedimentos, dependendo da idade, do tamanho e do peso do animal.

Com o tempo, a angiotomografia computadorizada (angio-TC) superou a angiografia convencional em muitos cenários, devido às suas diversas vantagens, como a economia e a rapidez para mobilizar profissionais necessários para execução. Também é menos invasiva e mórbida e, por isso, representa a modalidade de escolha para o sistema circulatório em diversas situações ${ }^{28}$. No entanto, descrições da anatomia vascular suína por tomografia são limitadas ${ }^{5}$.

\section{Considerações sobre modelos de treinamento e pesquisa em procedimentos vasculares}

\section{a) Acesso vascular}

O acesso vascular, utilizando a técnica de Seldinger, pode ser realizado por punção guiada por ultrassonografia ou dissecção cirúrgica ${ }^{1}$. A dissecção da artéria femoral é realizada na artéria femoral superficial e não na femoral comum, devido ao fato de esta ser intrapélvica nos suínos. Durante a dissecção da carótida comum, devem-se evitar lesões da cadeia simpática, localizada dentro da bainha carotídea, medial e dorsal ao nervo vago, o que pode resultar em síndrome de Horner. Vasodilatadores locais podem ser necessários durante a manipulação do território carotídeo, pois o espasmo ocorre com facilidade ${ }^{1}$. Assim como nos seres humanos, a relação próxima entre o esôfago e a artéria carótida comum esquerda deve ser lembrada.

\section{b) Coagulação}

Comparados aos humanos, suínos apresentam hipercoagulabilidade, tornando a anticoagulação intraoperatória muito importante ${ }^{1,30}$. Como na prática clínica, administra-se heparina intravenosa (100-300 unidades $/ \mathrm{kg}$ ), mantendo o tempo de coagulação ativado $\geq 250 \mathrm{~s}^{7}$. Dependendo do procedimento (implante de stent, bypass com próteses, 
por exemplo), complementa-se a terapia antitrombótica com ácido acetilsalicílico e/ou clopidogrel no período pós-operatório ${ }^{1,11}$

\section{c) Stents}

O diâmetro deve ser apropriado ao vaso no qual serão implantados, com sobredimensionamento mínimo (1,0 a 1,1 vezes o diâmetro do vaso), para evitar que lesões mecânicas por dilatação excessiva gerem dados não fidedignos sobre segurança e eficácia, enquanto que stents muito pequenos podem migrar $^{1}$. Resultados apresentados nas Tabelas 1 e 3 podem auxiliar na escolha do diâmetro dos stents.

As artérias devem ser cuidadosamente selecionadas, para que os stents sejam implantados em vasos com características semelhantes às dos tratados na prática clínica. Isso é particularmente importante ao usar stents farmacológicos, pois vasos com características histológicas distintas podem responder de forma variada ao princípio ativo liberado ${ }^{1}$.

\section{d) Oclusão aórtica}

Na prática cirúrgica, a oclusão aórtica frequentemente é feita pela aplicação de pinças ou pela insuflação intraluminal de balões. Um estudo experimental em suínos, o qual incluiu análises histológicas e biomecânicas, revelou que os danos parietais e consequente diminuição da resistência aórtica são mais intensos quando a interrupção do fluxo aórtico é realizada com a aplicação de pinças do que quando é utilizada a oclusão intraluminal por balão ${ }^{31}$.

A circulação colateral exuberante explica porque a repercussão da oclusão arterial aguda pode ser dramaticamente diferente daquela observada em humanos. Em humanos, a oclusão aórtica aguda, em uma embolia a "cavaleiro", por exemplo, pode atingir $30 \%$ de mortalidade, enquanto que, em modelos suínos, foram encontrados vários casos oligossintomáticos de oclusão aguda e prolongada ${ }^{11}$.

Essas características sugerem que modelos suínos de oclusão aórtica aguda, embora anatomicamente adequados para o treinamento cirúrgico (como a REBOA, por exemplo), podem fornecer resultados imprecisos quanto à perspectiva fisiológica.

A técnica de REBOA evoluiu como alternativa ao clampeamento aórtico por toracotomia. Um balão complacente é introduzido na aorta e insuflado, obstruindo o fluxo para a circulação distal, aumentando a pós-carga e a pressão aórtica proximal, melhorando as perfusões miocárdica e cerebral com efeitos potencialmente benéficos em pacientes com choque hemorrágico profundo ${ }^{12,32,33}$
Modelos suínos têm sido utilizados para o desenvolvimento, treinamento e pesquisas sobre REBOA $^{12,29,32,33}$. As pesquisas enfocam comparações entre oclusão aórtica contínua e intermitente ${ }^{33-35}$, parcial versus total ${ }^{36}$, tempo ideal de oclusão ${ }^{37} \mathrm{e}$ oxigenação tecidual ${ }^{38}$, visando melhores resultados na prática clínica. Nossos resultados e os escassos dados da literatura sugerem que repercussões biológicas e histoquímicas da oclusão aórtica nos suínos devem ser criteriosamente interpretadas, pois o suprimento sanguíneo dos membros posteriores é assegurado por anastomoses, como as que existem entre as artérias torácica interna (mamária) e os vasos epigástricos ${ }^{11}$ (a Figura 4A demonstra o curso das artérias mamárias).

A rede colateral da parede abdominal inclui ainda as artérias ilíacas circunflexa profunda e lombares. Além disso, em caso de oclusão proximal, as proporções de diâmetro dos vasos sanguíneos envolvidos na perfusão dos membros posteriores favorecem muito mais a imediata perfusão colateral do que em humanos ${ }^{11}$. Embora não haja dúvida de que os modelos suínos sejam úteis para ensinar e praticar tais procedimentos cirúrgicos, essas particularidades podem tornar os resultados da oclusão da aorta infrarrenal não comparáveis aos obtidos na prática clínica.

\section{e) Procedimentos venosos}

Cateteres venosos são usualmente inseridos pela jugular externa, mais calibrosa do que a interna (Figura 2A), uma situação oposta à encontrada em humanos ${ }^{6,13,16}$.

Suínos têm sido utilizados para estudos sobre filtro de veia cava ${ }^{6,39,40}$. A punção do eixo femoral é possível; porém, recomenda-se que seja guiada por ultrassom, uma vez que a veia passa posteriormente à artéria, como observado na Figura 3D, dificultando a punção.

$\mathrm{O}$ modo como as veias hepáticas convergem para o segmento retro-hepático da veia cava caudal permite o treinamento de manobras usadas em cirurgia do trauma, como a de Pringle e a tríplice exclusão vascular hepática.

Após extensa busca na literatura, não foram encontrados artigos que descrevessem as situações análogas às síndromes de Nutcracker e May-Thurner em suínos, como as relatadas no presente estudo.

Durante a revisão bibliográfica, foi encontrado um estudo sobre o desenvolvimento de um stent venoso utilizando modelo animal ovino; entre as limitações mencionadas, estava o fato de que o stent havia sido implantado em uma veia sem estenose, pela dificuldade de simular tais condições em animais ${ }^{34}$; uma limitação potencialmente superada utilizando-se suínos cuja compressão venosa poderia ser detectada 
pré-experimento com exames não invasivos, conforme demonstrado no presente estudo.

Como a TC foi realizada com animais em decúbito ventral e devidamente hidratados, é improvável que o estreitamento descrito para as veias renal e ilíaca comum esquerdas (Figuras 2E e 2F) seja provocado pelo peso dos órgãos abdominais, sugerindo, de fato, um mecanismo mais semelhante ao encontrado em humanos. Estudos futuros podem se concentrar em examinar esses achados de estenose com ultrassom intravascular, ou em desenvolver modelos animais para implante de stents venosos.

Entre as limitações do estudo, encontram-se o fato de que apenas três animais foram estudados; também não foram estudados animais de ambos os sexos, pois os três animais eram machos.

\section{CONCLUSÕES}

Ao fornecer os dados aqui apresentados, este estudo pode facilitar o planejamento de ensaios préclínicos, as pesquisas básicas e o aperfeiçoamento do treinamento cirúrgico utilizando modelos suínos na área vascular.

\section{REFERÊNCIAS}

1. Crisóstomo V, Sun F, Maynar M, et al. Common swine models of cardiovascular disease for research and training. Lab Anim. 2016;45(2):67-74. http://dx.doi.org/10.1038/laban.935. PMid:26814353.

2. Mangla S, Choi JH, Barone FC, et al. Endovascular external carotid artery occlusion for brain selective targeting: a cerebrovascular swine model Neuroscience. BMC Res Notes. 2015;8(1):1-6. http:// dx.doi.org/10.1186/s13104-015-1714-7.

3. Siefert J, Hillebrandt $\mathrm{KH}$, Kluge $\mathrm{M}$, et al. Computed tomographybased survey of the vascular anatomy of the juvenile Göttingen minipig. Lab Anim. 2017;51(4):388-96. http://dx.doi. org/10.1177/0023677216680238. PMid:27932686.

4. Judge EP, Hughes JML, Egan JJ, Maguire M, Molloy EL, O'Dea S. Anatomy and bronchoscopy of the porcine lung: a model for translational respiratory medicine. Am J Respir Cell Mol Biol. 2014;51(3):334-43. http://dx.doi.org/10.1165/rcmb.2013-0453TR. PMid:24828366.

5. Bekheit M, Bucur PO, Wartenberg M, Vibert E. Computerized tomography-based anatomic description of the porcine liver. J Surg Res. 2017;210:223-30. http://dx.doi.org/10.1016/j.jss.2016.11.004. PMid:28457333.

6. Dondelinger RF, Ghysels MP, Brisbois D, et al. Relevant radiological anatomy of the pig as a training model in interventional radiology. Eur Radiol. 1998;8(7):1254-73. http://dx.doi.org/10.1007/ s003300050545. PMid:9724449.

7. Sakaoka A, Koshimizu M, Nakamura S, Matsumura K. Quantitative angiographic anatomy of the renal arteries and adjacent aorta in the swine for preclinical studies of intravascular catheterization devices. Exp Anim. 2018;67(2):291-9. http://dx.doi.org/10.1538/ expanim.17-0125. PMid:29353822.

8. Smith AC, Swindle MM. Preparation of Swine for the Laboratory ILAR J. 2006;47(4):358-63. http://dx.doi.org/10.1093/ilar.47.4.358. PMid:16963815.
9. Septimus Sisson SB. The anatomy of the domestic animals. 2nd ed. Philadelphia: W.B. Saunders Company; 1930. p. 734-42.

10. Michaud F, Li N, Plantefève R, et al. Selective embolization with magnetized microbeads using magnetic resonance navigation in a controlled-flow liver model. Med Phys. 2019;46(2):789-99. http://dx.doi.org/10.1002/mp.13298. PMid:30451303.

11. Haacke N, Unger JK, Haidenhein C, Russ M, Hiebl B, Niehues SM. Pig specific vascular anatomy allows acute infrarenal aortic occlusion without hind limb ischemia and stepwise occlusion without clinical signs. Clin Hemorheol Microcirc. 2011;48(1):17385. http://dx.doi.org/10.3233/CH-2011-1401. PMid:21876245.

12. Borger van der Burg BLS, Hörer TM, Eefting D, et al. Vascular access training for REBOA placement: a feasibility study in a live tissue-simulator hybrid porcine model. J R Army Med Corps. 2019;165(3):147-51. http://dx.doi.org/10.1136/jramc-2018-000972. PMid:30228195.

13. Florescu MC, Runge J, Lof J, et al. Surgical technique of placement of an external jugular tunneled hemodialysis catheter in a large pig model. J Vasc Access. 2018;19(5):473-6. http://dx.doi. org/10.1177/1129729818760964. PMid:29557223.

14. Kotsougiani D, Willems JI, Shin AY, Friedrich PF, Hundepool CA, Bishop AT. A new porcine vascularized tibial bone allotransplantation model: anatomy and surgical technique. Microsurgery. 2018;38(2):195202. PMid:29131389.

15. Kloster BO, Lund L, Lindholt JS. Laparo- and thoracoscopic aortic aneurysm neck optimization and treatment of potential endoleaks type IA and II in a porcine model. Ann Med Surg. 2015;5:5-10. http://dx.doi.org/10.1016/j.amsu.2015.11.002. PMid:26793311.

16. Swindle MM. Swine in the laboratory: surgery, anesthesia, imaging and experimental techniques. 2nd ed. Boca Raton: Taylor \& Francis Group; 2007. p. 214-52. http://dx.doi.org/10.1201/9781420009156.

17. Genain MA, Morlet A, Herrtage $M$, et al. Comparative anatomy and angiography of the cardiac coronary venous system in four species: human, ovine, porcine, and canine. J Vet Cardiol. 2018;20(1):33-44. http://dx.doi.org/10.1016/j.jvc.2017.10.004. PMid:29191414.

18. Habib CA, Utriainen D, Peduzzi-Nelson J, et al. MR imaging of the yucatan pig head and neck vasculature. J Magn Reson Imaging. 2013;38(3):641-9. http://dx.doi.org/10.1002/jmri.24003. PMid:23348984.

19. Kim SH. Doppler US and CT diagnosis of nutcracker syndrome. Korean J Radiol. 2019;20(12):1627. http://dx.doi.org/10.3348/ kjr.2019.0084. PMid:31854150.

20. Sankaran L, Ramachandran R, Bala Raghu Raji V, Periasamy Varadaraju P, Panneerselvam P, Radhakrishnan PR. The role of multidetector CT angiography in characterizing vascular compression syndromes of the abdômen. Egypt J Radiol Nucl Med. 2019;50(1):1-12. http:// dx.doi.org/10.1186/s43055-019-0063-2.

21. Sablón González N, Lorenzo Villalba N, Parodis López Y, González Díaz P, Boada Díaz J, Kechida M. Nutcracker syndrome. Medicina. 2019;79(2):150-3. PMid:31048282

22. White JM, Comerota AJ. Venous compression syndromes. Vasc Endovascular Surg. 2017;51(3):155-68. http://dx.doi. org/10.1177/1538574417697208. PMid:28330436.

23. Jayaraj A, Buck W, Knight A, Johns B, Raju S. Impact of degree of stenosis in May-Thurner syndrome on iliac vein stenting. J Vasc Surg Venous Lymphat Disord. 2019;7(2):195-202. http://dx.doi. org/10.1016/j.jvsv.2018.10.001. PMid:30553783.

24. Kunio M, Wong G, Markham PM, Edelman ER. Sex differences in the outcomes of stent implantation in mini-swine model. PLoS One. 2018;13(1):e0192004. http://dx.doi.org/10.1371/journal. pone.0192004. PMid:29377941. 
25. von Trotha KT, Butz N, Grommes J, et al. Vascular anatomy of the small intestine - a comparative anatomic study on humans and pigs. Int J Colorectal Dis. 2015;30(5):683-90. http://dx.doi. org/10.1007/s00384-015-2163-4. PMid:25694139.

26. Yoshida RA, Yoshida WB, Rollo HA, Kolvenbach R, Lorena SERS. Curva de aprendizado em cirurgia aórtica videolaparoscópica: estudo experimental em porcos. J Vasc Bras. 2008;7(3):231-8. http://dx.doi.org/10.1590/S1677-54492008000300008.

27. Nickel R, Schummer E, Seiferle E. The anatomy of the domestic animals: the circulatory system, the skin, and the cutaneous organs of the domestic mammals. Berlin: Springer-Verlag; 1981. p. 72-73. (vol. 3).

28. Rosow DE, Sahani D, Strobel O, et al. Imaging of acute mesenteric ischemia using multidetector $\mathrm{CT}$ and $\mathrm{CT}$ angiography in a porcine model. J Gastrointest Surg. 2005;9(9):1262-75. http://dx.doi. org/10.1016/j.gassur.2005.07.034. PMid:16332482.

29. White JM, Cannon JW, Stannard A, Markov NP, Spencer JR, Rasmussen TE. Endovascular balloon occlusion of the aorta is superior to resuscitative thoracotomy with aortic clamping in a porcine model of hemorrhagic shock. Surgery. 2011;150(3):400-9. http://dx.doi.org/10.1016/j.surg.2011.06.010. PMid:21878225.

30. Chen Y, Tillman B, Go C, et al. A novel customizable stent graft that contains a stretchable ePTFE with a laser-welded nitinol stent. J Biomed Mater Res B Appl Biomater. 2019;107(4):911-23. http:// dx.doi.org/10.1002/jbm.b.34186. PMid:30176119.

31. Prata MP, Jaldin RG, Lourenção PLTA, et al. Lesão aguda da parede arterial provocada pelo método de interrupção temporária de fluxo em diferentes vias de cirurgia aórtica: estudo morfológico e biomecânico da aorta de porcos. J Vasc Bras. 2020;19:e20190025. http://dx.doi.org/10.1590/1677-5449.190025. PMid:32499823.

32. Smith SA, MCAlister VC. A novel REBOA system: prototype and proof of concept. Can J Surg. 2018;6(6):188-94. PMid:30417720.

33. Borger van der Burg BLS, van Schaik J, Brouwers JJWM, et al. Migration of Aortic Occlusion Balloons in an in vitro model of the human circulation. Injury. 2019;50(2):286-91. http://dx.doi. org/10.1016/j.injury.2018.12.026. PMid:30594315.

34. Che H, Jiang J, Liu H, Wei J, Zhang X. Preliminary report of a new type of braided vein stent in animals. Phlebology. 2019;34(4):24656. http://dx.doi.org/10.1177/0268355518801172. PMid:30227789.

35. Kuckelman JP, Barron M, Moe D, et al. Extending the golden hour for Zone 1 resuscitative endovascular balloon occlusion of the aorta: Improved survival and reperfusion injury with intermittent versus continuous resuscitative endovascular balloon occlusion of the aorta of the aorta in a porcine severe truncal hemorrhage model. J Trauma Acute Care Surg. 2018;85(2):318-26. http://dx.doi. org/10.1097/TA.0000000000001964. PMid:30080780.

36. Reva VA, Matsumura Y, Samokhvalov IM, et al. Defining degree of aortic occlusion for partial-REBOA: a computed tomography study on large animals. Injury. 2018;49(6):1058-63. http://dx.doi. org/10.1016/j.injury.2018.04.021. PMid:29699730.

37. Reva VA, Matsumura Y, Hörer T, et al. Resuscitative endovascular balloon occlusion of the aorta: what is the optimum occlusion time in an ovine model of hemorrhagic shock? Eur J Trauma Emerg
Surg. 2018;44(4):511-8. http://dx.doi.org/10.1007/s00068-0160732-z. PMid:27738726.

38. Lam JH, O'Sullivan TD, Park TS, et al. Non-invasive dual-channel broadband diffuse optical spectroscopy of massive hemorrhage and Resuscitative Endovascular Balloon Occlusion of the Aorta (REBOA) in swine. Mil Med. 2018;183(Suppl 1):150-6. http:// dx.doi.org/10.1093/milmed/usx163. PMid:29635570.

39. Eggers MD, McArthur MJ, Figueira TA, et al. Pilot in vivo study of an absorbable polydioxanone vena cava filter. J Vasc Surg Venous Lymphat Disord. 2015;3(4):409-20. http://dx.doi.org/10.1016/j. jvsv.2015.03.004. PMid:26992619.

40. Wang Z, Ashley DW, Kong L, Kang J, Nakayama DK, Dale PS. Nuclear factor- $\kappa B$ is activated in filter-implanted vena cava. Cardiovasc Intervent Radiol. 2019;42(4):601-7. http://dx.doi.org/10.1007/ s00270-018-2138-2. PMid:30535787.

Correspondência Adenauer Marinho de Oliveira Góes Junior Rua Domingos Marreiros, 307/ 802 CEP 66055-210 - Belém (PA), Brasil Tel.: (91) 98127-9656 E-mail: adenauerjunior@gmail.com

Informações sobre os autores AMOG) - Professor de cirurgia vascular, Centro Universitário do Estado do Pará (CESUPA); Aluno de Pós-doutorado, Programa de Ciência Cirúrgica Interdisciplinar, Universidade Federal de São Paulo (UNIFESP).

RHFC - Professora de habilidades cirúrgicas, Centro Universitário do Estado do Pará (CESUPA); Médica veterinária; Doutora em Saúde e Produção Animal na Amazônia, Universidade Federal Rural da Amazônia (UFRA).

IPF - Professora de bioestatística; Farmacêutica, Centro Universitário do Estado do Pará (CESUPA); Doutora em Biologia Parasitária na Amazônia (PPGBPA/UEPA/IEC).

EMR, FBAA e JHAS - Estudantes de medicina, Centro Universitário do Estado do Pará (CESUPA)

CPO - Médica egressa, Centro Universitário do Estado do Pará (CESUPA).

SCVA - Professora Adjunta Livre-Docente, Departamento de Cirurgia, Universidade Federal de São Paulo (UNIFESP); Orientadora, Programa de Ciência Cirúrgica Interdisciplinar, Universidade Federal de São Paulo (UNIFESP).

Contribuições dos autores Concepção e desenho do estudo: AMOGJ, SCVA Análise e interpretação dos dados: AMOGJ, SCVA, RHFC, IPF Coleta de dados: AMOGJ, RHFC, CPO, EMR Redação do artigo: AMOG), FBAA, CPO Revisão crítica do texto: AMOG], SCVA, RHFC, IPF, FBAA, EMR, JHAS, CPO

Aprovação final do artigo*: AMOGJ, SCVA, RHFC, IPF, FBAA, EMR, JHAS, CPO Análise estatística: IPF Responsabilidade geral pelo estudo: AMOG)

*Todos os autores leram e aprovaram a versão final submetida do J Vasc Bras. 